\title{
A DIFFERENTIAL EQUATION OF LURIE TYPE
}

\author{
T. A. BURTON
}

ABSTRACT. The problem of Lurie consists of finding necessary and sufficient conditions for all solutions of the system $\left\{x^{\prime}=\right.$ $\left.A x+b f(\sigma), \quad \sigma^{\prime}=c^{x} x-r f(\sigma)\right\}$ to tend to zero as $t \rightarrow \infty$ under appropriate conditions on the functions involved. When $f(\sigma) / \sigma \rightarrow 0$ as $|\sigma| \rightarrow \infty$, we obtain NASC's for all solutions to be uniformly ultimately bounded.

1. Introduction. We consider a system of real ordinary differential equations

$$
\begin{aligned}
& x^{\prime}=A x+b f(\sigma), \quad,=d / d t, \\
& \sigma^{\prime}=c^{T} x-r f(\sigma),
\end{aligned}
$$

in which $f:(-\infty, \infty) \rightarrow(-\infty, \infty)$ is sectionally continuous with $\sigma f(\sigma)>0$ for $\sigma \neq 0, A$ is an $n \times n$ constant matrix whose characteristic roots have negative real parts, $c$ and $b$ are constant $n$-vectors, and $r$ is a positive constant. The problem of Lurie consists of finding necessary and sufficient conditions in order that every solution $(\sigma(t), x(t))$ of $(1)$ tend to $(0,0)$ as $t \rightarrow \infty$ (cf. [5, p. 9]). A very good summary of the work done on the problem is given by Lefschetz [5] who devotes the entire monograph to the problem. The treatment by Aiserman and Gantmacher [1] is also excellent and is somewhat closer to our work as they assume that $0<f(\sigma) / \sigma \leqq M$ for some constant $M$. In fact, the problem is one from control theory, but it may be treated simply as a system of ordinary differential equations.

The major advances on the problem deal with finding NASC's for certain Liapunov functions to be positive definite with negative definite derivatives (cf. [5, p. 22]). This then yields conditions for bounded solutions to tend to zero. However, independent arguments have been needed to show that solutions are bounded [3]. In addition, these approaches must be criticized on the grounds that NASC's for the specified Liapunov function to be positive definite with a negative definite derivative do not

Received by the editors April 1, 1972.

AMS 1969 subject classifications. Primary 3451; Secondary 3440.

Key words and phrases. Problem of Lurie, differential equation, boundedness of solutions, control theory.

(c) American Mathematical Society 1973 
necessarily imply NASC's for the problem of Lurie as the Liapunov function may be poorly chosen. It appears to the author that the problem of Lurie itself is generally untouched as we can find no NASC for all solutions of (1) to tend to zero under any nonlinear restrictions on $f$.

In order to make an inroad we restrict the control function $f$ so that it satisfies $f(\sigma) / \sigma \rightarrow 0$ as $|\sigma| \rightarrow \infty$ and show that all solutions are bounded if and only if $c^{T} A^{-1} b+r \geqq 0$. It then follows easily that solutions are uniformly ultimately bounded if and only if $c^{T} A^{-1} b+r>0$. This is not, of course, the solution of the problem of Lurie; but it is a truly significant step in that direction.

2. Boundedness. We now give our main result.

THEOREM. Let the characteristic roots of $A$ have negative real parts, $\sigma f(\sigma)>0$ if $\sigma \neq 0, f(\sigma) / \sigma \rightarrow 0$ as $|\sigma| \rightarrow \infty$, and let $r>0$. All solutions of (1) are bounded if and only if $c^{T} A^{-1} b+r \geqq 0$.

Proof. As the characteristic roots of $A$ have negative real parts, there is a positive definite matrix $B$ such that $A^{T} B+B A=-I$ (cf. $[2, \mathrm{p}$. 295]). Define a function $V_{1}$ by $V_{1}(\sigma, x)=x^{T} B x$ so that along any solution $(\sigma(t), x(t))$ of (1) we have

$$
V_{1}^{\prime}=-x^{T} x+b^{T} f(\sigma) B x+x^{T} B b f(\sigma) \leqq-x^{T} x+m|x||f(\sigma)|
$$

for some $m>0$.

We now specify certain numbers which will be used in finding a region $U$ having special properties and in which $V_{1}^{\prime} \leqq 0$. There are positive numbers $k, L, K_{1}$, and $K_{2}$ such that $\left|c^{T} A^{-1} x\right| \leqq L|x|, K_{2}|x|^{2} \leqq\left|x^{T} B x\right| \leqq$ $K_{1}|x|^{2}$, and $k<\min \left\{1,\left(K_{2}\right)^{1 / 2} / 3 L\right\}$.

For $|\sigma| \geqq 1$, we have $V_{1}^{\prime} \leqq-x^{T} x+m|x||\sigma||f(\sigma) / \sigma|$ and so if $\left(x^{T} B x\right)^{1 / 2} \geqq$ $k|\sigma|$, then there exists $R>0$ such that $|\sigma| \geqq R$ implies $V_{1}^{\prime} \leqq 0$. Also, as $V_{1}^{\prime} \leqq-x^{T} x+m|x||f(\sigma)|$, if $|\sigma| \leqq R$, then there is an $\bar{R}$ such that $|x| \geqq \bar{R}$ implies that $V_{1}^{\prime} \leqq 0$. Thus, if we let $H=1+R+\bar{R}$, then on $U=$ $\left\{(\sigma, x)\left|\left(x^{T} B x\right)^{1 / 2} \geqq k\right| \sigma \mid\right.$ and $\left.|(\sigma, x)| \geqq H\right\}$ we have $V_{1}^{\prime} \leqq 0$.

We now define another function, $V_{2}$, and show that for properly chosen constants the intersection of the sets defined by $V_{1}=c_{1}$ and $V_{2}=c_{2}$ lies in $U$.

Let $V_{2}(\sigma, x)=\left(c^{T} A^{-1} x-\sigma\right)^{2} / 2$ and notice that from the first equation in (1) we may obtain

$$
c^{T} A^{-1} x^{\prime}=c^{T} x+c^{T} A^{-1} b f(\sigma)
$$

which, with the second equation in (1), yields

$$
c^{T} A^{-1} x^{\prime}-\sigma^{\prime}=\left(c^{T} A^{-1} b+r\right) f(\sigma)
$$


so that

$$
V_{2}^{\prime}=\left(c^{T} A^{-1} x-\sigma\right)\left(c^{T} A^{-1} b+r\right) f(\sigma) .
$$

(This derivative had previously been noted by La Salle [3].) (At this point, the reader is advised to make a sketch of the $\sigma-c^{T} A^{-1} x$-plane and note the properties of $V_{2}=$ constant and the sign of $V_{2}^{\prime}$ when $|\sigma|>\left|c^{T} A^{-1} x\right|$.)

Let $c_{1}>0$ be given with $c_{1}$ large as will be specified later. We seek $c_{2}$ such that $\left\{(\sigma, x) \mid c^{T} A^{-1} x-\sigma= \pm\left(2 c_{2}\right)^{1 / 2}\right.$ and $\left.x^{T} B x=c_{1}\right\}$ is contained in $\left\{(\sigma, x)\left|\left(x^{T} B x\right)^{1 / 2}>k\right| \sigma \mid\right\}$ so that for large $c_{1}$ the intersection of $V_{1}=c_{1}$ with $V_{2}=c_{2}$ will be in $U$. To this end, note that

$$
\begin{aligned}
|\sigma| & =\left|c^{T} A^{-1} x \mp\left(2 c_{2}\right)^{1 / 2}\right| \leqq L|x|+\left(2 c_{2}\right)^{1 / 2} \\
& \leqq\left(L /\left(K_{2}\right)^{1 / 2}\right)\left(x^{T} B x\right)^{1 / 2}+\left(2 c_{2}\right)^{1 / 2}=\left(L /\left(K_{2}\right)^{1 / 2}\right)\left(c_{1}\right)^{1 / 2}+\left(2 c_{2}\right)^{1 / 2} .
\end{aligned}
$$

Thus, we want

$$
|\sigma| \leqq L\left(c_{1} / K_{2}\right)^{1 / 2}+\left(2 c_{2}\right)^{1 / 2}<\left(\left(c_{1}\right)^{1 / 2}\right) / k=\left(\left(x^{T} B x\right)^{1 / 2}\right) / k,
$$

so we need to pick $c_{2}$ so that $\left(2 c_{2}\right)^{1 / 2}<\left(c_{1}\right)^{1 / 2}\left([1 / k]-\left[L /\left(K_{2}\right)^{1 / 2}\right]\right)$. As $k<\left(K_{2}\right)^{1 / 2} / L$, we define $M$ by $(1 / k)-\left(L /\left(K_{2}\right)^{1 / 2}\right)=(4 M)^{1 / 2}$ so that $\left(2 c_{2}\right)^{1 / 2}<$ $\left(4 M c_{1}\right)^{1 / 2}$. Then $c_{2}=M c_{1}$ is a satisfactory choice for $c_{2}$. (C. E. Langenhop assisted with this paragraph.)

Now $x^{T} B x=c_{1}$ implies that $|x| \leqq\left(c_{1} / K_{2}\right)^{1 / 2}$ and $|x| \geqq\left(c_{1} / K_{1}\right)^{1 / 2}$. Then $\sigma=c^{T} A^{-1} x+\left(2 c_{2}\right)^{1 / 2}$ implies that

$$
\begin{aligned}
|\sigma| & \geqq-L\left(c_{1} / K_{2}\right)^{1 / 2}+\left(2 c_{2}\right)^{1 / 2}=-L\left(c_{1} / K_{2}\right)^{1 / 2}+\left(2 M c_{1}\right)^{1 / 2} \\
& =\left(c_{1}\right)^{1 / 2}\left[(2 M)^{1 / 2}-\left(L /\left(K_{2}\right)^{1 / 2}\right)\right] .
\end{aligned}
$$

Now $(2 M)^{1 / 2}>L /\left(K_{2}\right)^{1 / 2}$ if and only if $(4 M)^{1 / 2}>L 2^{1 / 2} /\left(K_{2}\right)^{1 / 2}$ if and only if $(1 / k)-\left(L /\left(K_{2}\right)^{1 / 2}\right)>L 2^{1 / 2} /\left(K_{2}\right)^{1 / 2}$ if and only if $1 / k>L\left(1+2^{1 / 2}\right) /\left(K_{2}\right)^{1 / 2}$ if and only if $k<\left(K_{2}\right)^{1 / 2} /\left[L\left(1+2^{1 / 2}\right)\right]$, and the last inequality is implied by a condition on the choice of $k$. A similar result is obtained for $\sigma=$ $c^{T} A^{-1} x-\left(2 c_{2}\right)^{1 / 2}$. Thus, for $c_{1}$ sufficiently large, we see that the intersection of $V_{1}=c_{1}$ and $V_{2}=c_{2}$ yields $|\sigma|>H$ and $|x|>H$. Thus, the intersection lies in $U$ and $\sigma$ does not vanish.

Next, note that the set of points $(\sigma, x)$ satisfying $V_{1}(\sigma, x) \leqq c_{1}$ and $V_{2}(\sigma, x) \leqq c_{2}$, for given positive constants $c_{1}$ and $c_{2}$, is bounded. We call this set $W\left(c_{1}, c_{2}\right)$. Notice also that for each point $\left(\sigma_{1}, x_{1}\right)$ outside $W\left(c_{1}, c_{2}\right)$, we may choose $\left(c_{1}^{*}, c_{2}^{*}\right)$ with $\left(\sigma_{1}, x_{1}\right)$ in the boundary of $W\left(c_{1}^{*}, c_{2}^{*}\right)$ and $W\left(c_{1}, c_{2}\right) \subset W\left(c_{1}^{*}, c_{2}^{*}\right)$.

The sufficiency of our condition will now be shown. Assume that $c^{T} A^{-1} b+r \geqq 0$. Let $\left(\sigma_{0}, x_{0}\right)$ be given and let $W\left(c_{1}, c_{2}\right)$ be chosen containing $\left(\sigma_{0}, x_{0}\right)$ with $c_{2}=M c_{1}$ and with its boundary outside $|(\sigma, x)| \leqq H$. Denote by $(\sigma(t), x(t))$ a solution of (1) through $\left(\sigma_{0}, x_{0}\right)$. Notice that 
$(\sigma(t), x(t))$ does not leave $W\left(c_{1}, c_{2}\right)$ along $V_{2}=c_{2}$, since $V_{2}^{\prime} \leqq 0$ on this part of the boundary of $W\left(c_{1}, c_{2}\right)$ and so $V_{2}$ would be increasing along the solution. (Note that the part of the boundary given by $V_{2}=c_{2}$ satisfies $\sigma>c^{T} A^{-1} x$ with $\sigma>0$ and $\sigma<c^{T} A^{-1} x$ with $\sigma<0$.) Also, $(\sigma(t), x(t))$ does not leave $W\left(c_{1}, c_{2}\right)$ along $V_{1}=c_{1}$ for the same reason, since the intersection of $V_{1}=c_{1}$ with $V_{2}=c_{2}$ is in $U$. Thus, $(\sigma(t), x(t))$ is bounded.

We now suppose that $c^{T} A^{-1} b+r<0$ and show that there are unbounded solutions. Let $(\sigma(t), x(t))$ be a solution of (1) satisfying $x(0)=0$ and $\sigma(0)>0$ and so large that for $c_{2}>0$ defined by $\sigma^{2}(0)=2 c_{2}$ and $c_{1}$ defined by $c_{2}=M c_{1}$, then the boundary of $W\left(c_{1}, c_{2}\right)$ lies outside $|(\sigma, x)| \leqq H$. At $t=0$, we have $V_{2}^{\prime}>0$ so that $c^{T} A^{-1} x(t)-\sigma(t) \leqq-\left(2 c_{2}\right)^{1 / 2}$ for $t$ close to zero, but $t$ positive. From $V_{2}^{\prime}=\left(c^{T} A^{-1} x-\sigma\right)\left(c^{T} A^{-1} b+r\right) f(\sigma)$, we see that $V_{2}^{\prime}>0$ as long as $\sigma(t)>0$.

If $\sigma(t)>0$ for all $t \geqq 0$, then $V_{2}(\sigma(t), x(t))$ is defined for all $t \geqq 0$ and $V_{2}^{\prime}$ will remain positive as $c^{T} A^{-1} x(t)-\sigma(t)$ will remain negative. Thus, it will follow from La Salle's theorem (cf. [4, p. 62]) that $(\sigma(t), x(t))$ is unbounded.

We therefore suppose that there is a $t_{2}>0$ such that $\sigma\left(t_{2}\right)=0$ and $\sigma(t)>0$ for $0 \leqq t<t_{2}$. It is then possible to find $t_{1}$ satisfying $0<t_{1}<t_{2}$ such that $\left(x^{T}\left(t_{1}\right) B x\left(t_{1}\right)\right)^{1 / 2}=k \sigma\left(t_{1}\right)$ and $\left(x^{T}(t) B x(t)\right)^{1 / 2}>k \sigma(t)$ for $t_{1}<t \leqq t_{2}$. Thus, on $\left(t_{1}, t_{2}\right)$ we have $V_{1}^{\prime} \leqq 0$ and $V_{2}^{\prime}>0$. Therefore, for $t_{1} \leqq t \leqq t_{2}$, we have

and so

$$
K_{2}|x(t)|^{2} \leqq x^{T}(t) B x(t) \leqq x^{T}\left(t_{1}\right) B x\left(t_{1}\right)=k^{2} \sigma^{2}\left(t_{1}\right)
$$

$$
\left|x\left(t_{2}\right)\right| \leqq k \sigma\left(t_{1}\right) /\left(K_{2}\right)^{1 / 2} .
$$

Also, for $t_{1} \leqq t \leqq t_{2}$ we have $V_{2}^{\prime} \geqq 0$ so

$$
\left(c^{T} A^{-1} x(t)-\sigma(t)\right)^{2} \geqq\left(c^{T} A^{-1} x\left(t_{1}\right)-\sigma\left(t_{1}\right)\right)^{2},
$$

and if $\sigma\left(t_{2}\right)=0$, then $\left|c^{T} A^{-1} x\left(t_{2}\right)\right| \geqq\left|c^{T} A^{-1} x\left(t_{1}\right)-\sigma\left(t_{1}\right)\right|$, and so

$$
L\left|x\left(t_{2}\right)\right| \geqq\left|c^{T} A^{-1} x\left(t_{2}\right)\right| \geqq\left|c^{T} A^{-1} x\left(t_{1}\right)-\sigma\left(t_{1}\right)\right| \geqq \sigma\left(t_{1}\right)-\left|c^{T} A^{-1} x\left(t_{1}\right)\right|
$$

or

$$
L\left(\left|x\left(t_{2}\right)\right|+\left|x\left(t_{1}\right)\right|\right) \geqq \sigma\left(t_{1}\right) .
$$

But $k \sigma\left(t_{1}\right)=\left(x^{T}\left(t_{1}\right) B x\left(t_{1}\right)\right)^{1 / 2} \geqq\left(K_{2}\left|x\left(t_{1}\right)\right|^{2}\right)^{1 / 2}$ and so $\left|x\left(t_{1}\right)\right| \leqq k \sigma\left(t_{1}\right) /\left(K_{2}\right)^{1 / 2}$. Thus,

and so

$$
L\left(\left|x\left(t_{2}\right)\right|+k \sigma\left(t_{1}\right) /\left(K_{2}\right)^{1 / 2}\right) \geqq L\left(\left|x\left(t_{2}\right)\right|+\left|x\left(t_{1}\right)\right|\right) \geqq \sigma\left(t_{1}\right)
$$

$$
\left|x\left(t_{2}\right)\right| \geqq \sigma\left(t_{1}\right)\left[1-L k /\left(K_{2}\right)^{1 / 2}\right] / L .
$$

Then $(\alpha)$ and $(\beta)$ yield

$$
k \sigma\left(t_{1}\right) /\left(K_{2}\right)^{1 / 2} \geqq\left|x\left(t_{2}\right)\right| \geqq \sigma\left(t_{1}\right)\left[1-L k /\left(K_{2}\right)^{1 / 2}\right] / L
$$


or $k \geqq\left(K_{2}\right)^{1 / 2} / 2 L$. As $k<\left(K_{2}\right)^{1 / 2} / 3 L$, we have a contradiction. This completes the proof.

REMARK 1. Let $c_{1}$ and $c_{2}$ be related by $c_{2}=M c_{1}$ with $c_{1}$ so large that the boundary of $W\left(c_{1}, c_{2}\right)$ is outside $|(\sigma, x)| \leqq H$. We have already noticed that if $c_{2}^{*}=M c_{1}^{*}$ and $c_{1}^{*}>c_{1}$, then $W\left(c_{1}, c_{2}\right) \subset W\left(c_{1}^{*}, c_{2}^{*}\right)$. Also, when $c^{T} A^{-1} b+r>0$, then $V_{1}^{\prime}$ or $V_{2}^{\prime}$ is negative on those parts of the boundary of $W\left(c_{1}^{*}, c_{2}^{*}\right)$ consisting of $V_{1}=c_{1}^{*}$ or $V_{2}=c_{2}^{*}$ respectively. Thus, it is evident that $c^{T} A^{-1} b+r>0$ is a necessary and sufficient condition for solutions of (1) to be uniformly ultimately bounded.

REMARK 2. One of the difficulties encountered by previous investigators is the problem of showing boundedness of solutions when $\int_{0}^{\sigma} f(s) d s$ is bounded (cf. [3, p. 601]). Our results indicate that this condition has nothing to do with the problem.

The following is a typical result of previous investigators. Lurie used the Liapunov function

$$
V(\sigma, x)=x^{T} B x+\int_{0}^{\sigma} f(s) d s,
$$

where $B$ is positive definite. Lefschetz then showed that

$$
V^{\prime}=-x^{T} D x+f(\sigma)\left[2 b^{T} B+c^{T}\right] x-r f^{2}(\sigma),
$$

with $D=-\left(A^{T} B+B A\right)$, will be negative definite if and only if $D$ is positive definite and

$$
r>(B b+c / 2)^{T} D^{-1}(B b+c / 2) .
$$

To that point, it could not be concluded that solutions of (1) were bounded unless $\int_{0}^{\sigma} f(s) d s \rightarrow \infty$ as $|\sigma| \rightarrow \infty$, thereby making $V$ radially unbounded. However, La Salle showed that $(\gamma)$ alone was sufficient for boundedness. Furthermore, La Salle showed that $(\gamma)$ implies $c^{T} A^{-1} b+r>0$, and this motivated our present work. (For more details, see La Salle [3].)

ADDED IN PROOF. A final remark should be added in the proof of the necessity of the condition $C^{T} A^{-1} b+r \geqq 0$ for boundedness. Although $C^{T} A^{-1} x(t)-\sigma(t)$ remains negative, $\sigma(t)$ could approach zero. But then $V_{1}(\sigma(t), x(t))$ would be defined for all $t$ past some $T$ and $V_{1}^{\prime}$ would be strictly negative. This would yield a contradiction.

\section{REFERENCES}

1. M. A. Aizerman and F. R. Gantmacher, Absolute stability of regulator systems, Izdat. Akad. Nauk SSSR, Moscow, 1963; English transl., Holden-Day, San Francisco, Calif., 1964; German transl., Oldenbourg Verlag, München-Wien, 1965. MR 32 \#1036.

2. J. K. Hale, Ordinary differential equations, Wiley, New York, 1969. 
3. J. P. La Salle, Complete stability of a nonlinear control system, Proc. Nat. Acad. Sci. U.S.A. 48 (1962), 600-603. MR 25 \#304.

4. - Stability theory for ordinary differential equations, J. Differential Equations 4 (1968), 57-65. MR 36 \#5454.

5. S. Lefschetz, Stability of nonlinear control systems, Math. in Sci. and Engineering, vol. 13, Academic Press, New York, 1965. MR 30 \#5860.

Department of Mathematics, Southern Illinois University, Carbondale, ILLINOIS 62901 